Proceedings of XIX International Scientific Conference "New Technologies and Achievements in Metallurgy, Material Engineering, Production Engineering and Physics", Częstochowa, Poland, June 7-8, 2018

\title{
Surface Morphology Analysis of Martensitic Stainless Steel after Different Treatments
}

\author{
M. GWoŹDziK ${ }^{a, *}$, S. Kulesza ${ }^{b}$, M. Bramowicz $^{c}$ And Z. BaŁaga ${ }^{a}$ \\ ${ }^{a}$ Częstochowa University of Technology, Faculty of Production Engineering and Materials Technology, \\ Institute of Materials Engineering, al. Armii Krajowej 19, 42-200 Częstochowa, Poland \\ ${ }^{b}$ University of Warmia and Mazury in Olsztyn, Faculty of Mathematics and Computer Science, \\ Department of Relativistic Physics, Olsztyn, Poland \\ ${ }^{c}$ University of Warmia and Mazury in Olsztyn, Faculty of Technical Sciences, \\ Department of Materials Technology and Machines, Olsztyn, Poland
}

\begin{abstract}
The paper presents research on fractal analysis of martensitic stainless steel used for the manufacturing of surgical instruments. Fractal analysis was performed on samples subjected to sequential processes: heat treatment, surface treatment, and sterilization. According to scanning electron microscopy images, the surface treatments end up in highly anisotropic surface texture composed of a bunch of straight, narrow ridges. Closer examination reveals that the ridges are actually chains of nearly identical spherical grains.
\end{abstract}

DOI: 10.12693/APhysPolA.135.157

PACS/topics: X39Cr13, SEM, fractal analysis, surface treatment

\section{Introduction}

Material engineering is a field of science used on a large scale [1-7], especially surface engineering [8-20]. Increasingly, attempts are made to modify the surface layer by various methods, and in particular nitriding is widely used $[9,10,12-20]$. In paper [8], the spray-formed Al alloy was selected for testing. Specimens were subject to surface treatment in the form of plasma nitriding with subsequent electron beam remelting. Researchers have shown that the AlN layer fitted perfectly to the surface of the electron beam remelting layer. Moreover, the metallurgical bonding was very well improved by the electron beam remelting process due to the elimination of the pores caused by nitriding. In paper [10] modification were made in $\mathrm{Al}$ alloy. On this alloy, the layer was prepared through the deposition of Ti film by magnetron sputtering ion plating system. After this, the plasma nitriding of titanium was used to coated on. The researchers have shown that the preparation of multiphase layer can remarkably improve the surface hardness and the wear rate for the multiphase layer decreases. In turn, Li et al. [9] conducted tests on martensitic steel, where the steel was treated by active screen plasma nitriding. In the paper it was shown that the anodic active screen plasma nitriding technique was found to be able to significantly improve the corrosion properties, hardness and wear resistance of AISI 420 martensitic stainless steel.

*corresponding author; e-mail: gwozdzik.monika@wip.pcz.pl

\section{Material and methods}

X39Cr13 martensitic stainless steel was selected for testing. The material was delivered in the form of soft annealed sheet (1 mm thick). Specimens were subjected to heat treatment (hardening - $\mathrm{H}$ ) and surface treatment in the form of plasma nitriding (hardening + nitriding - HN). The hardening was carried out at $T=1050^{\circ} \mathrm{C}$ for time $t=1200 \mathrm{~s}$. The nitriding was carried out at $T=460{ }^{\circ} \mathrm{C}$ and pressure $p=145 \mathrm{~Pa}$ for time $t=20 \mathrm{~h}$. Reactive atmosphere contained $25 \%$ of molecular nitrogen diluted with molecular hydrogen. Part of specimens was sterilized (hardening + nitriding + sterilization HNS). The sterilization by steam was carried out in an autoclave at $T=134{ }^{\circ} \mathrm{C}$ with pressure $p=0.21 \mathrm{MPa}$ for $t=0.5 \mathrm{~h}$ in four cycles.

Determination of characteristics of surface height variability of steel samples relies on processing SEM images in order to extract underlying statistical dependencies and non-random patterns in data series [21, 22]. Appropriate numerical routine involves multistep operations and begins with calculations of the autocorrelation $\operatorname{map} R$ :

$$
R_{m, n}=\frac{1}{2 S_{q}^{2}} \sum_{k=1}^{N-n} \sum_{l=1}^{N-m}(z(x+m, y+n) z(x, y)),
$$

where $(m, n)$ establishes discrete shift between original image and its lagged copy, $S_{q}$ - root-mean-square surface roughness, and $N$ - number of scan steps along each direction. Directional inhomogeneity in surface geometry can be expressed in terms of anisotropy ratio $S_{t r}$, defined as the ratio of extreme lateral correlations [23]:

$$
S_{t r}=\frac{L_{a 1}}{L_{a 2}}
$$


where $L_{a 1}$ and $L_{a 2}$ are the smallest and largest decay lengths, respectively. Likewise, half-widths at half maxima (HWHM) of the autocorrelation peak along these directions, denoted as $w_{a 1}$ and $w_{a 2}$, can be helpful in estimating specific bump size of dominant topographical features $d_{g}$ :

$$
d_{g}=w_{a 1}+w_{a 2} .
$$

In the next step, 2-dimensional autocorrelation function is averaged around its origin to obtain 1-dimensional autocorrelation function $R(\tau)$. This profile is directly turned into the structure function $S(\tau)$ through the formula [23]:

$$
S(\tau)=2 S_{q}^{2}(1-R(\tau)) .
$$

Sayles and Thomas demonstrated that the structure function is governed by specific scaling law [24]:

$$
S(\tau)=K \tau^{2(2-D)},
$$

where $D$ is the fractal dimension, and $K$ - pseudotopothesy. Sample plots of profile structure functions are shown in Fig. 1. In general, $D$ and $K$ correspond to the way, how the relative and absolute height variations maintain scale-invariance, respectively. However,

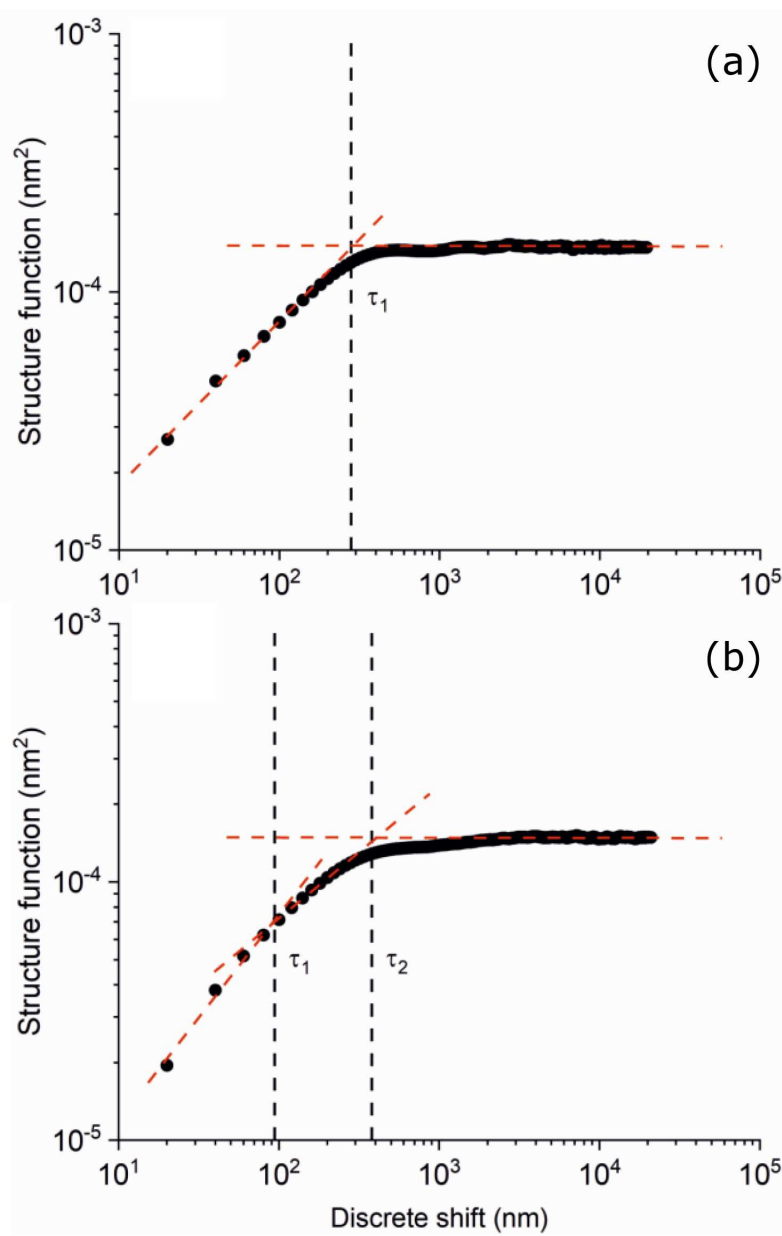

Fig. 1. Comparison of double-log plots of profile structure functions derived from SEM images of steel samples under study exhibiting different scaling characteristics: (a) monofractal and (b) bifractal.

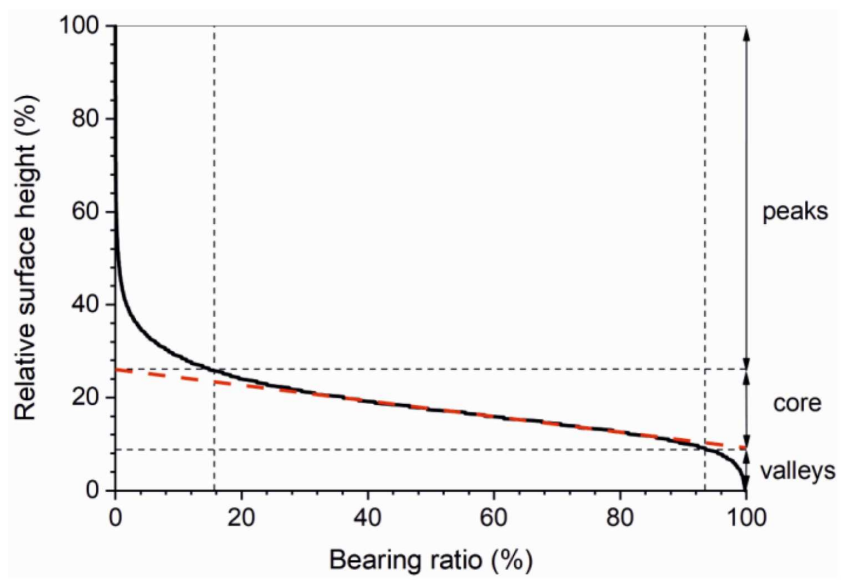

Fig. 2. Plot of the bearing curve illustrating the concept of estimation of valleys, core and peaks depths from SEM images.

the above allometricity breaks down beyond a certain threshold, referred to as the corner frequency $\tau_{c}$, at which the plot approaches constant level. On the other hand, in case of aggregated materials with higher-order alignment patterns, structure function follows two different scaling regimes describing single grains and clusters of grains independently of each other.

Apart from fractal parameters, the so-called functional descriptors of topographical complexity can be also derived using the bearing curve (Fig. 2). Actually, the curve represents cumulative distribution of relative surface heights plotted in a descending order. Among others, DIN 4776 standard specifies three main functional parameters:

a) core roughness depth $S_{k}$ - thickness of the flattest part of the bearing curve where the largest increase in surface height exists,

b) reduced peak height $S_{p k}$ - thickness of the bearing curve above the core profile,

c) reduced valley depth $S_{v k}$ - thickness of the bearing curve below the core profile.

\section{Result and discussion}

SEM images in Fig. 3 present intensity maps of steel samples in the digital domain. Each pixel on the grid corresponds to a discrete step on the sample with the value proportional to the signal intensity captured by the detector. Electron beam performs raster scans within rectangular area with sides of 64 by $44 \mu \mathrm{m}$. The images were taken after each step of the thermochemical treatment, that is: hardening $(\mathrm{H})$, hardening + nitriding $(\mathrm{HN})$ and hardening + nitriding + sterilization (HNS).

Analysis of the chemical composition (EDS) showed (Fig. 4) that the structure of the investigated steel after heat treatment is martensite with carbide precipitates, while after the surface treatment the surface layer was enriched with nitrogen. 

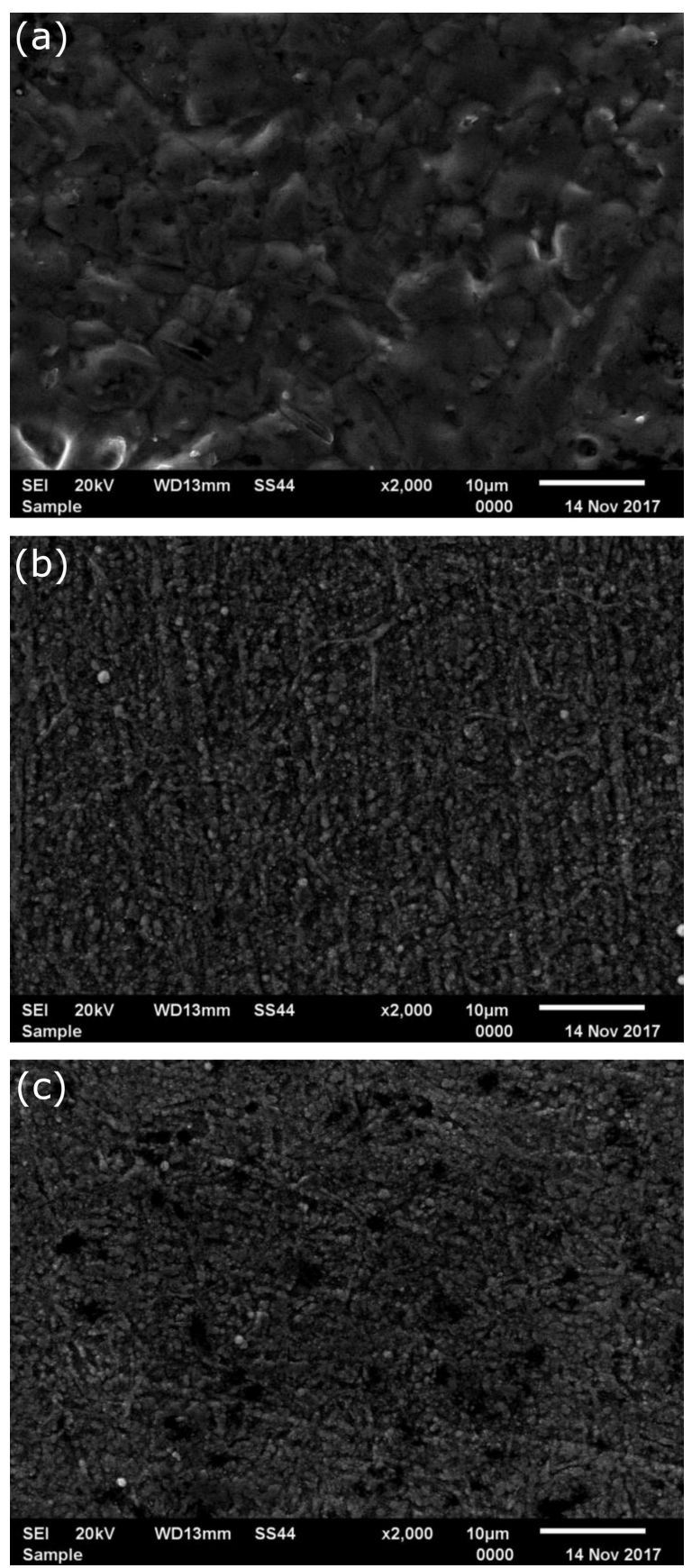

Fig. 3. SEM images of steel samples during multistep thermochemical treatment: (a) hardening $(\mathrm{H})$, (b) hardening + nitriding $(\mathrm{HN})$, (c) hardening + nitriding + sterilization (HNS).

Figure 3a reveals small, regular precipitates on otherwise rough surface of the steel specimen, each of which is several hundreds of nanometers in diameter. Although the precipitates appear randomly distributed over the surface, but more often than not they can be found next to the edges of much larger bumps of irregular shapes and few micrometers wide. The bumps exhibit no predominant axial alignment. Figure $3 \mathrm{~b}$ shows the surface of the specimen additionally subjected to the nitriding
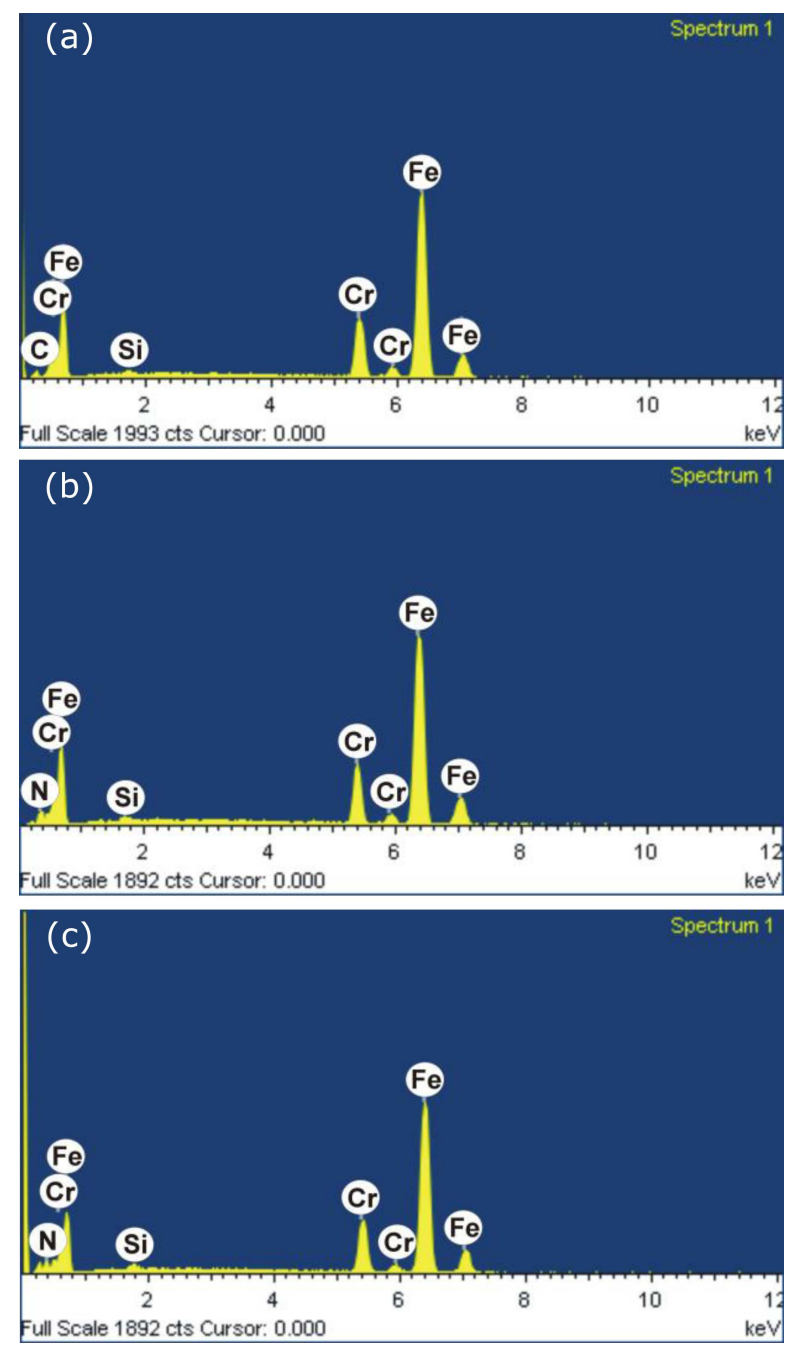

Fig. 4. SEM analysis of steel samples during multistep thermochemical treatment: (a) hardening $(\mathrm{H})$, (b) hardening + nitriding $(\mathrm{HN}),(\mathrm{c})$ hardening + nitriding + sterilization (HNS).

process. Unlike hardening, however, nitriding ends up in highly anisotropic surface texture composed of a bunch of straight, narrow ridges. Closer examination reveals that the ridges are actually chains of nearly identical spherical grains, which are around $750 \mathrm{~nm}$ in size, connected to each other within linear structures. Besides that, there are also another particles on the surface, which are 4-5 times smaller than the grains, well separated and randomly dispersed over the surface. Finally, Fig. 3c presents the surface of the sterilized steel sample, which is to a certain degree similar to the nitrided specimen. As in the previous image, there are numerous grains on the surface (about $750 \mathrm{~nm}$ in size) together with much smaller, $150 \mathrm{~nm}$ particles. However, even though the larger grains appear to be partly organized within shortorder linear structures, any longer ridges can be hardly found. Another difference is the presence of dimples on the surface of sterilized sample around $2-3 \mu \mathrm{m}$ wide, separated by $10-15 \mu \mathrm{m}$ from each other. 
TABLE I

Angle-dependent fractal metrics of surface geometry of steel samples along axes of extreme autocorrelation decays: $D$ - fractal dimension, $\tau-$ corner frequency, $a_{1}-$ slow-decay axis, $a_{2}$ - fast-decay axis.

\begin{tabular}{c|c|c|c|c|c|c|c|c}
\hline \hline \multirow{2}{*}{ Sample } & \multicolumn{4}{|c|}{$a_{1}$ axis } & \multicolumn{4}{c}{$a_{2}$ axis } \\
\cline { 2 - 9 } & $D_{1}$ & $\begin{array}{c}\tau_{1} \\
{[\mathrm{~nm}]}\end{array}$ & $D_{2}$ & $\begin{array}{c}\tau_{2} \\
{[\mathrm{~nm}]}\end{array}$ & $D_{1}$ & $\begin{array}{c}\tau_{1} \\
{[\mathrm{~nm}]}\end{array}$ & $D_{2}$ & $\begin{array}{c}\tau_{2} \\
{[\mathrm{~nm}]}\end{array}$ \\
\hline $\mathrm{H}$ & 1.86 & 130 & 1.67 & 1200 & 1.86 & 130 & 1.70 & 1600 \\
$\mathrm{HN}$ & 1.54 & 80 & 1.67 & 290 & 1.64 & 92 & 1.71 & 370 \\
$\mathrm{HNS}$ & - & - & 1.68 & 280 & 1.61 & 94 & 1.73 & 380
\end{tabular}

Table I presents angle-dependent fractal characteristics of the samples investigated in this study determined for directions of extreme decays of the autocorrelation function, that is $a_{1}$ and $a_{2}$. In general, all samples exhibit bifractal characteristics except for sterilized sample, which turns out to be monofractal along $a_{1}$ direction. Bifractality is widely agreed to be a fingerprint of aggregation process occurring on the surface with loworder fractal parameters describing the intracluster structures and the high-order ones related to the intercluster patterns. Among them, fractal dimension $D$ corresponds to the way how the relative height variations are invariant upon changes in the wavelength, and the corner frequency establishes a range in which such a scaling behavior occurs. Obtained fractal characteristics agree with predominant topographical features seen in SEM images.

As a rule, low-order fractal dimensions $D_{1}$ are found very large at 1.86 in all samples regardless of the observation angle, which is specific of well-developed surface structures, probably those of randomly distributed precipitates seen in Fig. 3a. However, after the nitriding and sterilization are carried out, this parameter sharply decreases to around 1.55-1.65, which in turn reflects changes towards less-developed surface structures. Unlike that, high-order fractal dimensions $D_{2}$ remain constant between 1.67 and 1.70 for both axes independent of the treatment procedure suggesting that complexity of intracluster structures do not vary at all. On the other hand, both low- and high-order corner frequencies $\tau_{1}$ and $\tau_{2}$, respectively, follow similar trends, although not to the same extent. Low-order corner frequency, likely associated with smaller particles seen in SEM images, equals to $130 \mathrm{~nm}$ in the hardened sample, but then decreases within 40 per cent to $80-90 \mathrm{~nm}$ in the nitrided and sterilized samples. Although $\tau_{1}$ is found consequently lower than the diameter of precipitates roughly by a factor of 2 , such a discrepancy might be eventually explained by irregularity in the shape of those particles. Likewise, high-order corner frequency somehow related to the dimension of the superstructures, is found very large at $1200-1600 \mathrm{~nm}$ in the hardened sample, but only 280-380 $\mathrm{nm}$ in the remaining ones in agreement with the changes seen in SEM images in Fig. 3. However, considering the fact that $\tau_{2}$ in the latter samples takes only a half of the size of the grains forming linear patterns, one might conclude that these chains are not that similar as they appear in pictures.

Table II presents spatial characteristics of the same steel specimens, derived by angular averaging of the autocorrelation maps around their origins. The averaging was aimed to help determine more general characteristics of the height variations, for example the size of dominant bumps on the surface. Table II shows that this number equals to $1000 \mathrm{~nm}$ in the hardened sample, but declines after nitriding by a factor of 4 . In comparison with dimensions of specific surface structures seen in SEM images, obtained results turn out to be roughly underestimated by a factor of 3 .

\section{TABLE II}

Average metrics of surface geometry of steel samples: $d_{g}$ - grain size, $S_{t r}$ - texture anisotropy ratio, $D$ fractal dimension, $\tau$ - corner frequency, $S_{k}$ - core roughness depth, $S_{p k}$ - reduced peak height, $S_{v k}$ - reduced valley depth.

\begin{tabular}{c|c|c|c|c|c|c|c|c|c}
\hline \hline Sample & $\begin{array}{c}d_{g} \\
{[\mathrm{~nm}]}\end{array}$ & $S_{t r}$ & $D_{1}$ & $\begin{array}{c}\tau_{1} \\
{[\mathrm{~nm}]}\end{array}$ & $D_{2}$ & $\begin{array}{c}\tau_{2} \\
{[\mathrm{~nm}]}\end{array}$ & $\begin{array}{c}S_{k} \\
{[\%]}\end{array}$ & $\begin{array}{c}S_{p k} \\
{[\%]}\end{array}$ & $\begin{array}{c}S_{v k} \\
{[\%]}\end{array}$ \\
\hline $\mathrm{H}$ & 1000 & 0.52 & 2.90 & 110 & 2.69 & 1300 & 17 & 74 & 9.2 \\
$\mathrm{HN}$ & 240 & 0.70 & 2.60 & 110 & 2.74 & 370 & 29 & 66 & 4.3 \\
$\mathrm{HNS}$ & 210 & 0.80 & 2.64 & 100 & 2.79 & 410 & 26 & 73 & 0.20
\end{tabular}

Another discrepancy between averaged spatial characteristics and main features displayed in the images can be found considering anisotropy ratio $S_{t r}$. According to SEM images, it is the nitrided sample, which is expected to be the most anisotropic due to aligned ridges, while the hardened sample is likely the most isotropic because of irregular, homogeneously distributed grains. Surprisingly, the hardened sample is found the most anisotropic and the sterilized one the most isotropic in terms of $S_{t r}$, which may be explained by the fact that the correlation lengths generally decrease in the nitrided and sterilized samples compared with the hardened one as indicated by decrease in the high-order corner frequency and grain dimension. Hence, to maintain the anisotropy ratio, these lengths need to shorten proportionally, which is hardly likely.

Trends in fractal dimensions of various orders are found quite opposite. Low-order fractal dimension decreases from 2.90 to 2.64 from hardened to sterilized sampled, whereas the high-order fractal dimension actually increases, which might be due to the apparent waviness not filtered out in the nitrided and sterilized samples. The size of the agglomerated particles expressed by the corner frequency $\tau_{1}$ remain constant at around $100 \mathrm{~nm}$ regardless of the treatment, but the size of the clusters defined by $\tau_{2}$ goes roughly hand in hand with the grain dimension.

Each of the functional parameters behaves in a different manner. Relative core roughness depth $S_{k}$, which defines the part of the surface profile bearing the largest loading, increases from 17 to $26-29$ per cent during the 
treatment. On the other hand, reduced peak height $S_{p k}$, which is worn at the earliest during normal loading, remains constant at around 70 per cent. Finally, reduced valley depth $S_{v k}$ responsible for carrying lubricants and other fluids, sharply vanishes from 9 per cent to zero. Note, however, that obtained results might be somehow biased by the linearization procedure used to generate pseudo-3D surface maps from plane SEM images.

\section{Summary}

On the basis of the obtained research results, the following statements and conclusions were formulated:

- After heat treatment there were observed small, regular precipitates on otherwise rough surface of the steel specimen, each of which is several hundreds of nanometers in diameter.

- After surface treatment the research showed the anisotropic surface texture composed of spherical grains $(750 \mathrm{~nm}$ in size), connected to each other within linear structures. Besides that, there are also another particles on the surface, which are 45 times smaller than the grains, well separated and randomly dispersed over the surface.

- After sterilization there are also numerous grains on the surface (about $750 \mathrm{~nm}$ in size) together with much smaller, $150 \mathrm{~nm}$ particles. But the larger grains do not create the ridges like after surface treatment. After sterilization there are also dimples on the sample around $2-3 \mu \mathrm{m}$ wide, separated by 10-15 $\mu \mathrm{m}$ from each other.

\section{References}

[1] S. Bose, D. Ke, H. Sahasrabudhe, A. Bandyopadhyay, Prog. Mater Sci. 93, 45 (2018).

[2] A. Jung, A. Buchwalder, E. Hegelmann, P. Hengst, R. Zenker, Surf. Coat. Technol. 335, 166 (2018).

[3] Y. Li, Y. He, J. Xiu, W. Wang, Y. Zhu, B. Hu, Surf. Coat. Technol. 329, 184 (2017).

[4] C. Lu, J.W. Yao, Y.X. Wang, Y.D. Zhu, J.H. Guo, Y. Wang, H.Y. Fu, Z.B. Chen, M.F. Yan, Appl. Surf. Sci. 431, 32 (2018).
[5] W.R. de Oliveira, B.C.E.S. Kurelo, D.G. Ditzel, F.C. Serbena, C.E. Foerster, G.B. de Souza, Appl. Surf. Sci. 434, 1161 (2018).

[6] R. Selvabharathi, R. Muralikannan, Mater. Sci. Eng. A 709, 232 (2018).

[7] K.L.M. Taaca, M.R. Vasquez Jr., Appl. Surf. Sci. 432, 324 (2018).

[8] L.A. Espitia, H. Dong, X.-Y. Li, C.E. Pinedo, A.P. Tschiptschin, Wear 376-377, 30 (2017).

[9] R. Kumar, R. Singh, D. Hui, L. Feo, F. Fraternali, Composites Part B 134, 193 (2018).

[10] K. Lin, X. Li, H. Dong, P. Guo, D. Gu, Vacuum 148 , 224 (2018).

[11] Q. Luo, O. Oluwafemi, M. Kitchen, S. Yang, Wear 376-377, 1640 (2017).

[12] A. Samanta, M. Bhattacharya, I. Ratha, H. Chakraborty, S. Datta, J. Ghosh, S. Bysakh, M. Sreemany, R. Rane, A. Joseph, S. Mukherjee, B. Kundu, M. Das, A.K. Mukhopadhyay, J. Mech. Behav. Biomed. Mater. 77, 267 (2018).

[13] G. Sharma, B. Thakur, M. Naushad, A. Kumar, F.J. Stadler, S.M. Alfadul, G.T. Mola, Environ. Chem. Lett. 16, 113 (2018).

[14] E. Zdravecká, J. Slota, P. Solfronk, M. Kolnerová, J. Mater. Eng. Perform. 26, 3588 (2017).

[15] G.-H. Zhao, R. Aune, N. Espallargas, J. Mech. Behav. Biomed. Mater. 63, 100 (2016).

[16] A.F. Yetim, M. Yazici, J. Bionic Eng. 11, 620 (2014).

[17] B.C.E.S. Kurelo, W.R. de Oliveira, F.C. Serbena, G.B. de Souza, Surf. Coat. Technol. 353, 199 (2018).

[18] L. Tang, W.J. Jia, J. Hu, Mater. Lett. 231, 91 (2108).

[19] P. Snopiński, T. Tański, K. Labisz, S. Rusz, P. Jonsta, M. Król, Int. J. Mater. Res. 107, 637 (2016).

[20] M. Krupiński, B. Krupińska, Z. Rdzawski, K. Labisz, T. Tański, J. Therm. Anal. Calorim. 120, 1573 (2015).

[21] M. Gwoździk, M. Bramowicz, S. Kulesza, in: Proc. 27th Int. Conf. on Metallurgy and Materials (METAL 2018), Brno (Czech Republic), 2018, p. 1114.

[22] N. Naseri, S. Solaymani, A. Ghaderi, M. Bramowicz, S. Kulesza, Ş. Ţălu, M. Pourreza, S. Ghasemi, $R S C$ Adv. 7, 12923 (2017).

[23] W.P. Dong, P.J. Sullivan, K.J. Stout, Wear 178, 45 (1994).

[24] R.S. Sayles, T.R. Thomas, Wear 42, 263 (1977). 